\title{
Performance of geometrical effect in wavelength filtrate detection using 10Gbps data rate for free space optical communication system
}

\author{
M. F. Talib, Anuar M. S., C. B. M. Rashidi \\ Advance Communication Engineering, Centre of Excellence (ACE-COE), \\ School of Computer and Communication Engineering, University Malaysia Perlis (UniMAP), Perlis, Malaysia
}

\begin{tabular}{l} 
Article Info \\
\hline Article history: \\
Received Sep 27, 2018 \\
Revised Nov 25, 2018 \\
Accepted Dec 8, 2018 \\
\hline
\end{tabular}

Keywords:

Aperture receiver

Beam divergence

BER

Free space optic (FSO)

Power received

\begin{abstract}
In this paper, the analysis of comparison between proposed detection called as Wavelength Filaztrate Detection with existing detection have been analyzed to determine the best system performance in term of power received and bit error rate (BER). The analysis focus on the geometrical effect of aperture receiver and beam divergence. The performance of Free Space Optics is validated through simulation analysis using parameter 10Gbps data rate and in $14 \mathrm{~dB} / \mathrm{km}$ atmospheric attenuation. This analysis shows that Wavelength Filtrate Detection increase the power received of $6 \mathrm{dBm}$ and improve the absolute value of error rate 10-9 in acceptable threshold required by (ITR-U) as much as $50 \%$ compare to others existing detection.
\end{abstract}

Copyright $\odot 2019$ Institute of Advanced Engineering and Science. All rights reserved.

\section{Corresponding Author:}

M. F. Talib,

Advance Communication Engineering, Centre of Excellence (ACE-COE),

School of Computer and Communication Engineering,

University Malaysia Perlis (UniMAP), Perlis, Malaysia.

Email: fauzan.talib@gmail.com

\section{INTRODUCTION}

Free Space Optical (FSO) communication system is a flexible system contains laser beam acts as the medium to transmit the signal through the atmosphere [1]. This interesting system can achieve high bandwidth of data with a lower cost. The system structure is quite easily to handle and currently carry large amount of data widely best suited in multi-Gbps communication. The performance remains relatively simple which transfer using optical signal from transmission station one point and successively received to other point receiver station. This system applied such as in building to building, satellite to ground, aircraft to ground and ship to ship [2], [3]. Low power consumption and require only small space make the system easily to install. Therefore, no licence is needed from Federal Communications Commission (FCC) to setup this FSO system. High atmospheric attenuation likes heavy rain cause the signal attenuated and degrade the system performance. So, to avoid this bad line of sight (LOS) in FSO system, the receiver detection should more sensitive which can operate in a poor weather condition. This analysis investigates the performance of Dual Diffuser Modulation/Wavelength Filtrate Detection technique based on simulation result of bit error rate $(\mathrm{BER})$ and power received.

\section{GEOMETRICAL LOSSES}

Figure 1 shows the typical FSO block diagram that include the transmitter and receiver parts. The geometric losses are the losses that occur due to spreading of the transmitted beam divergence between 
the transmitter and receiver. The geometric losses are simply the ratio of the surface area of the receive aperture to the surface area of the transmit beam divergence at the receiver. The geometric losses depend primarily on the divergence and the range. Therefore, the geometrical losses for receiver detection are the main factor to know the performance of signal losses is higher or lower based on error rate and power received [4].

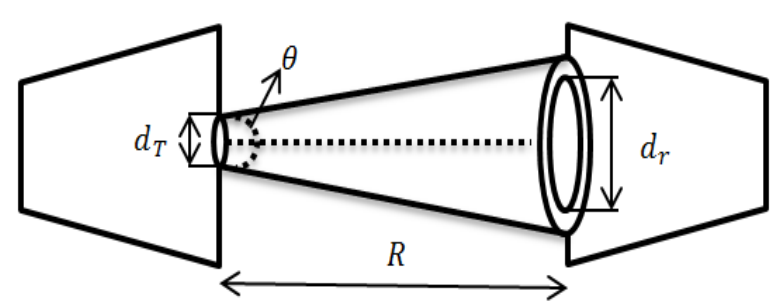

Figure 1. Typical free space optics block diagram

$$
\frac{d_{Y}{ }^{3}}{\left(d_{T}+(\theta R)\right)^{2}}
$$

where: $d_{y}=$ radius of aperture receiver

$d_{T}=$ radius of aperture transmitter

$\theta=$ beam divergence

$R=$ range

\section{SIMULATION DESIGN}

The Optisystem software can be used to design and simulate the performance of optical network to find the bit error rate and power received. The design layout for FSO system contains transmitter part, atmospheric channel and receiver part [5].

\subsection{Transmitter}

In this subsystem, the data are generated in the Pseudo Random Bit Generator (PRBS). The characteristics of data sequence are design as a random bit. The binary output of 1 and 0 represent in this data sequence will encode in the Non-Return-to-Zero (NRZ) generator. The optical signal that came from Continuous Wave (CW) laser and electrical pulse generator will sends to the Mach-Zehnder Modulator to carry the signal to the FSO link [6].

\subsection{Channel}

Free Space Optical (FSO) channel is a tool that uses a principle of laser beam from transmitter through the atmosphere into receiver. This subsystem contains two telescopes. The diameter aperture sizes are different in transmitter and receiver. The size of beam divergence also determine the state in this free space channel [7].

\subsection{Receiver}

In this part, some of the incoming signals with a noise bins transmit to the filters Fibre Bragg Grating (FBG) and then to the Photodetector PIN. The photodetector convert light (optical signal) into current (electrical signal) and when the photon already absorbed sufficient energy, it can generate the current. The 3R Regenerator generates the original bit sequence and regulated electrical signal to be utilized for BER examination. Finally, the shape of eye diagram can display in the BER Analyser and the value of power received in the electrical power meter visualizer [8].

\section{DUAL DIFFUSER MODULATION TECHNIQUE}

This design technique is named as Dual Diffuser Modulation (DDM) as illustrated in in Figure 2 which is upgraded based on the conventional technique that is On Off Keying (OOK). The magnitude of signal is double compare to the original technique where it's containing an original signal and inversion 
signal. The signal will transmit to the receive1r station for differential detection process. These two signals will go through the subtractor which subtracts the original signal and inversion signal as shown in Figure 2. So, the output signal become maximum ' 1 ' and minimum ' -1 '. These modification techniques increase the threshold detection and can improve the power received with a better BER value [9], [10].

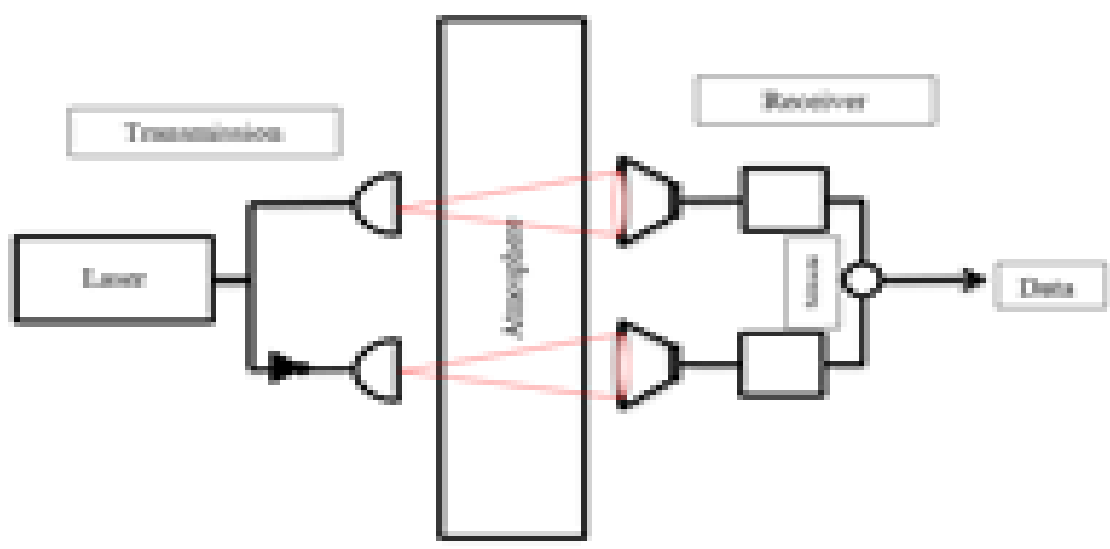

Figure 2. DDM technique in FSO system setup [10]

\section{RECEIVER DETECTION}

In FSO communication system, the detection process affects the design of transceiver. In this section, the analysis focus on receiver detection to find the performance of signal received. The explanation about design of receiver detection are presented as follows.

\subsection{Subtractor Detection}

The subtractor detection explains that the signal received is divided into two. This detection does not use a Fibre Bragg Grating (FBG) sensor at design detection. The first photodetector received the original signal and the second photodetector received the inversion signal. Then, the signal was combined in subtractor which subtracts the first signal and second signal as shown in Figure 3. The signal will have a positive and negative amplitudes which the magnitude of signal can reaching double [4].

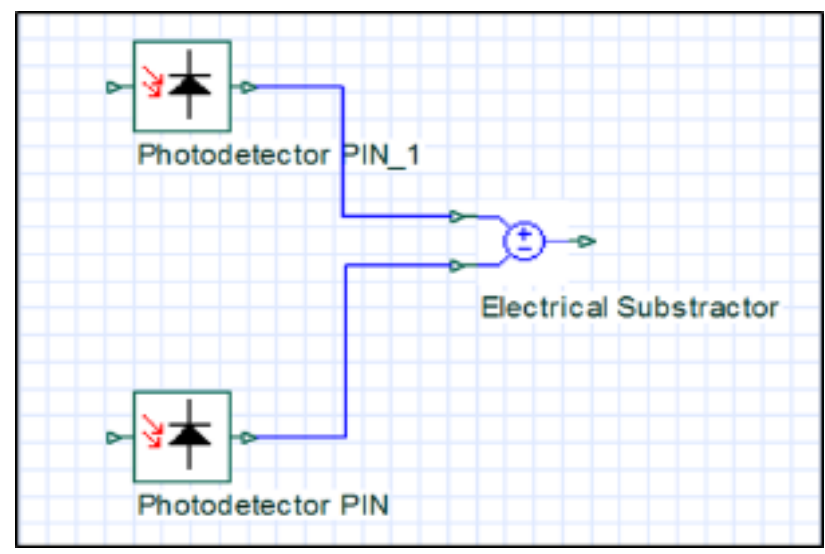

Figure 3. Subtractor detection design

\subsection{Spectral Direct Detection}

This detection technique represents the implementation of Fibre Bragg Grating (FBG) whereby just a pair of decoder and detector is required as illustarated in Figure 4. For this detection design, the spectral chip is requiring in the optical domain when it already filtered. This detection does not bother with subtraction detection technique at the electrical part. In this way, there will be no subtractor used to subtract 
the covering data. Along these lines the quantity of filters is fundamentally decreased $[11,12]$. It is clear that the component being directly detected at the receiver side and hence its design is simple and less costly.

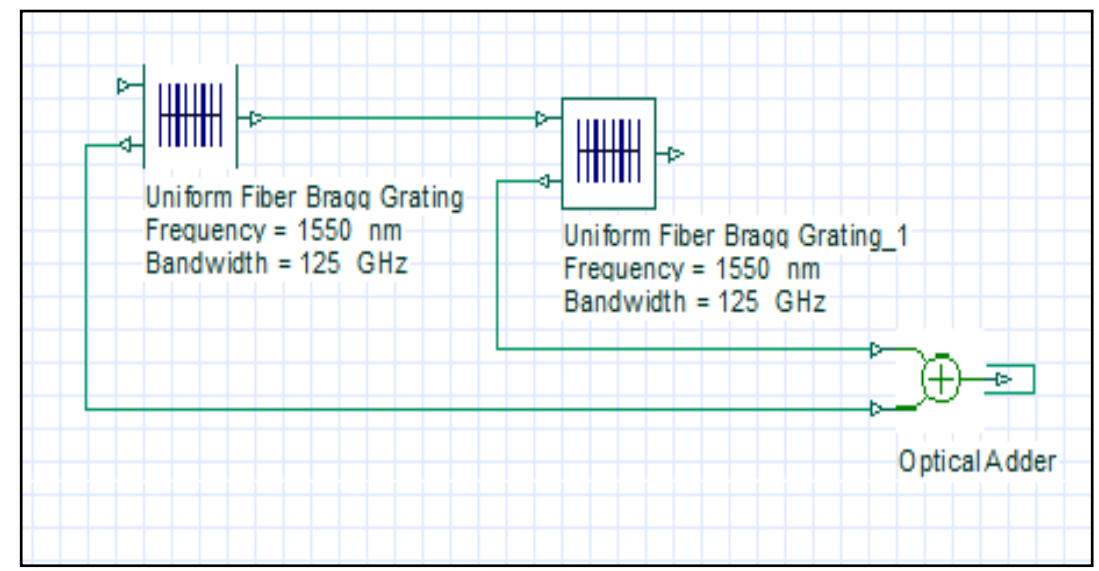

Figure 4. Spectral direct detection

\subsection{Complimentary Detection}

This detection design is divided into two complementary parts of spectral chips. The upper part is a decoder, which is spectrally identical to an encoder which used to extract the signal encoded on the transmitter station. The lower part contains a complementary decoder which is the logical complement of the decoder [13]. The two parts of signals are sent to a detector that computes the difference between both decoded signals as in Figure 5. The photodetectors will detect the combination of two signal which will be fed to the subtractor [14].

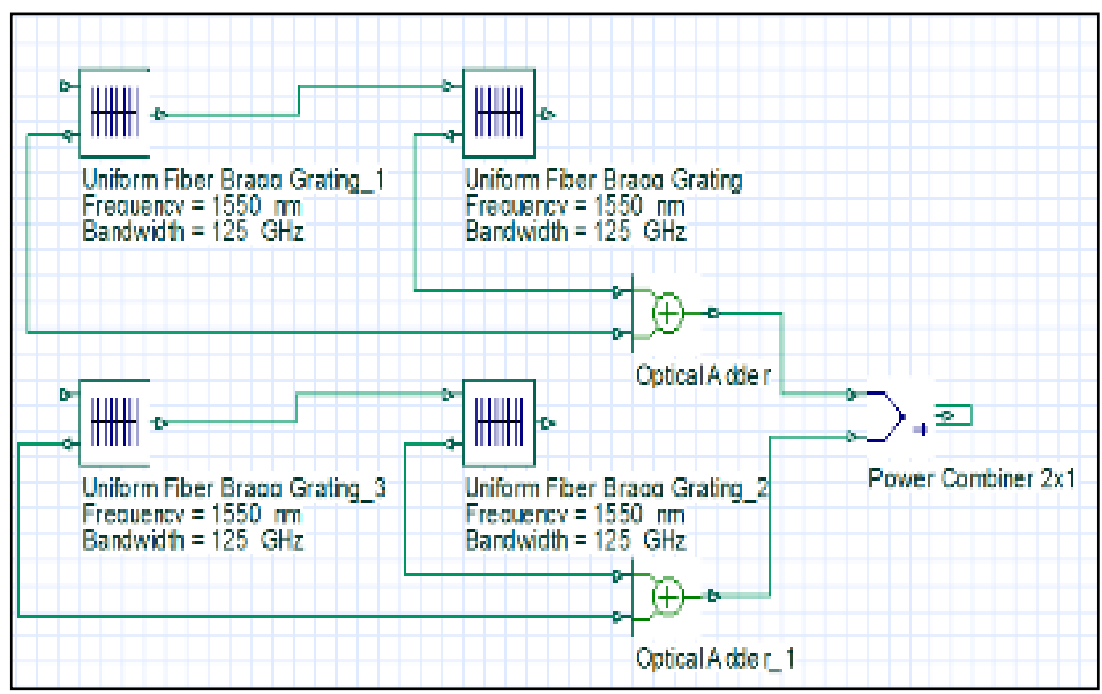

Figure 5. Complimentary detection design

\subsection{AND Detection}

The function of AND subtraction detection is it can cancel out the interference from channels. However, the design of detection is using a less number of filters in the decoder. In the receiver part, the input optical signal are splits into two parts, one to the decoder that has an identical filter structure with the encoder and the other to the decoder that has AND filter structures [15]. The two branches of spectral signals are sent to a detector to convert optical signal into electrical signal and then go through a subtractor used to subtract the overlapping data from the intended signal as depeicted in Figure 6. 


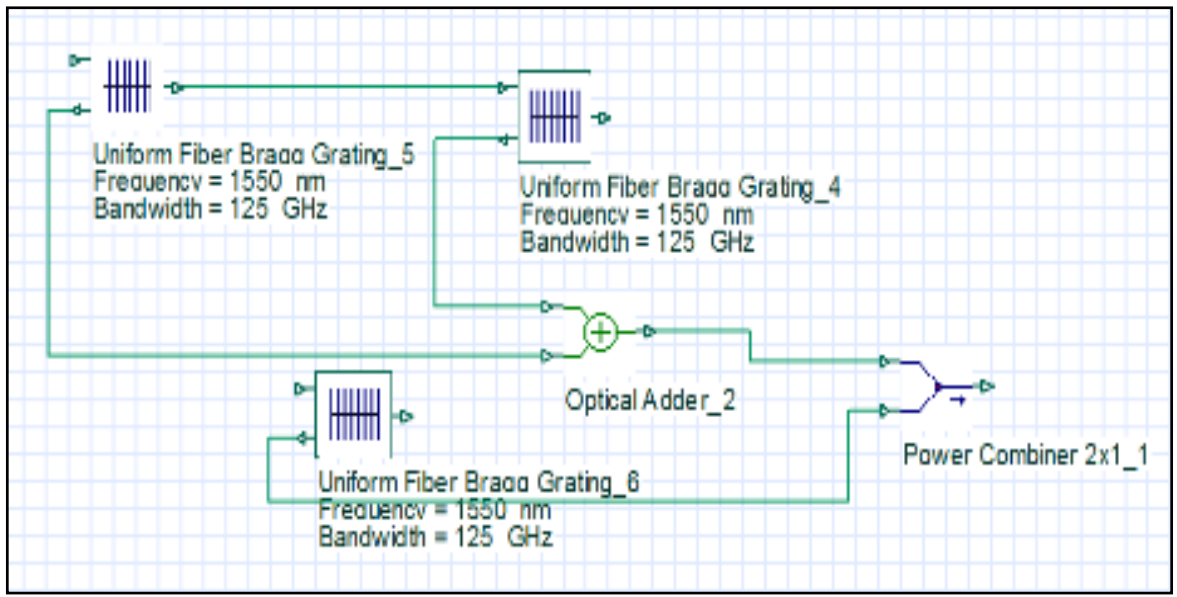

Figure 6. AND detection design

\subsection{Wavelength Filtrate Detection}

The proposed detection called as Wavelength Filtrate detection in FSO system. In simulation design, the received signal is split by fork into two parts that is upper and lower decoding branches. The decoders are placed in parallel configuration with a uniform spectral wavelength. The connection for this detection in fibre bragg grating sensor only used selective wavelength and neglected the other wavelength. After that the optical signal is combine upper and lower branch to go through the photodetector to provide an electrical signal from both decoders as in Figure 7. By using less number of fibre bragg grating, the power loss of this design can be reduced. Therefore, the complexity, cost and total power loss of the systems is reduced.

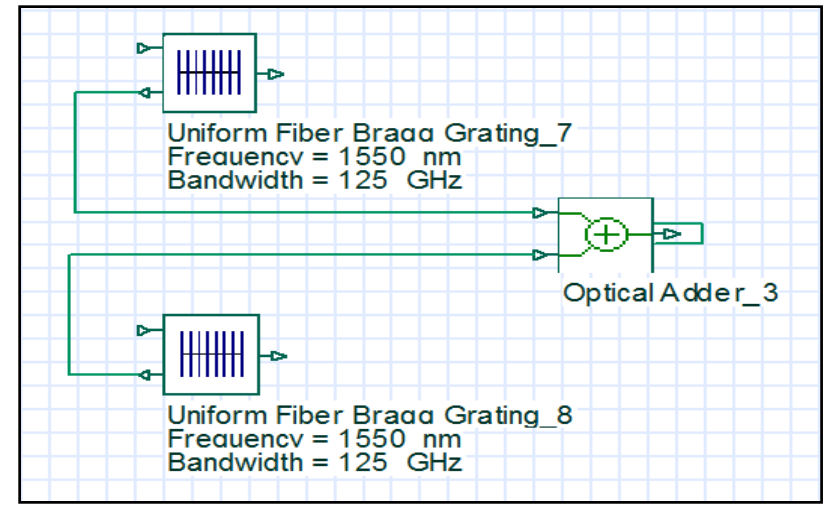

Figure 7. Wavelength filtrate detection design

\section{RESULT AND DISCUSSION}

The performance of BER and power received are carried out against the aperture receiver and beam divergence in a FSO system. Table 1 shows the parameter of simulation analysis using in this investigation.

Table 1. Parameter of FSO System

\begin{tabular}{cc}
\hline Parameters & Value $^{\mathrm{a}}$ \\
\hline Data Rate & $10 \mathrm{Gbps}$ \\
Wavelength & $1550 \mathrm{~nm}$ \\
Power Transmit & $0 \mathrm{dBm}$ \\
Range & $1 \mathrm{~km}$ \\
Aperture Transmitter & $5 \mathrm{~cm}$ \\
& \\
Attenuation & $14 \mathrm{~dB} / \mathrm{km}$ \\
\hline
\end{tabular}


This Figure 8 explains about the bit error rate versus aperture receiver for selection receiver detection. The size of aperture needs to be carefully chosen to avoid high signal losses when the bad atmospheric effect occurs. It is truly proved that, the DDM_WFD support small aperture size $51 \mathrm{~cm}$ as well as capable to detect good signal compare to DDM_Subtractor that using bigger aperture size $98 \mathrm{~cm}$ to achieve standard BER 10-9. The increasing of $41 \mathrm{~cm}$ aperture size in DDM_Subtractor make the cost of lens will expensive to buy. For DDM_Complimentary, DDM_AND and DDM_SDD accommodate the aperture size of $64 \mathrm{~cm}, 70 \mathrm{~cm}$ and $93 \mathrm{~cm}$ respectively.

From Figure 9, by referring to the $50 \mathrm{~cm}$ aperture receiver as a point, DDM_WFD received $-23 \mathrm{dBm}$ more power with a BER 3.65×10-9 compare to DDM_Subtractor that receive -29dBm power but the signal is totally loss. DDM_SDD also shows the signal totally loss when using $50 \mathrm{~cm}$ as an aperture receiver.

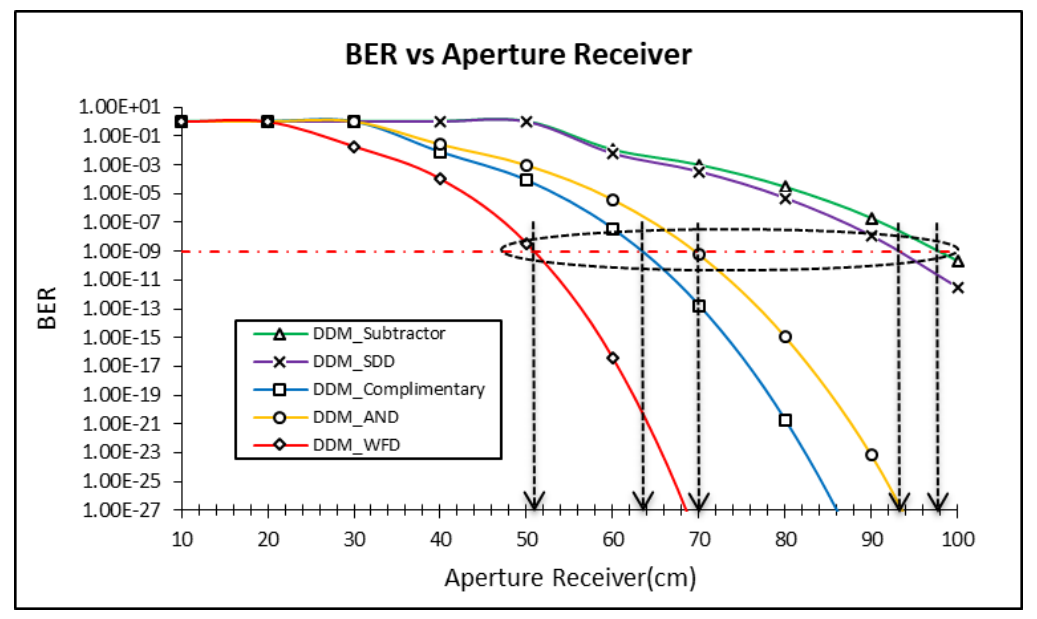

Figure 8. Bit error rate versus aperture receiver for selection receiver detection

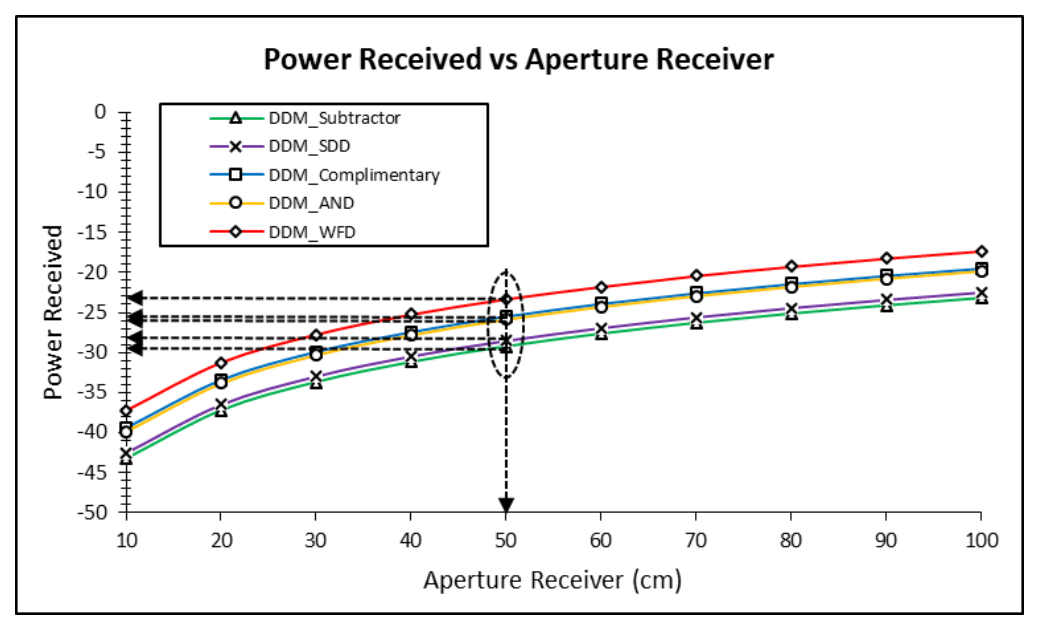

Figure 9. Power received versus aperture receiver for selection receiver detection

Meanwhile, Figure 10 shows the eye pattern of selective detection that explain about the level of losses in a situation of $50 \mathrm{~cm}$ aperture receiver. The effect for DDM_WFD is smoothly wide open eye compare to the other selective detection. This will make the performance of the system capable to reduce the noise effect.

Based on Figure 11, the performance of various beam divergence in FSO system versus BER is reflected. Typically, the optical beam width from a FSO transceiver will be relatively wide divergence, which is equivalent to a beam spread. This narrow beam allows for secure and efficient transmission with a major fraction of the transmitted power being collected by the receiver. According from the graph, in minimum BER 10-9 the DDM_WFD perform the maximum beam divergence available is $0.75 \mathrm{mrad}$ compare to the 
DDM_Subtractor, DDM_SDD, DDM_AND and DDM_Complimentary only available with 0.38mrad, $0.4 \mathrm{mrad}, 0.54 \mathrm{mrad}$ and $0.6 \mathrm{mrad}$ respectively. This detection improves the performance of signal received as well as can combat in a situation of adverse weather condition.

In the other hand, Figure 12 shows about the power received by referring $0.5 \mathrm{mrad}$ as a point. From this figure, the DDM_WFD can achieve an optimum value of power received -20dBm compare to DDM_Subtractor, DDM_SDD, DDM_Complimentary and DDM_AND detection only achieve -26dBm, $-25 \mathrm{dBm},-22 \mathrm{dBm}$ and $-22 \mathrm{dBm}$ respectively.

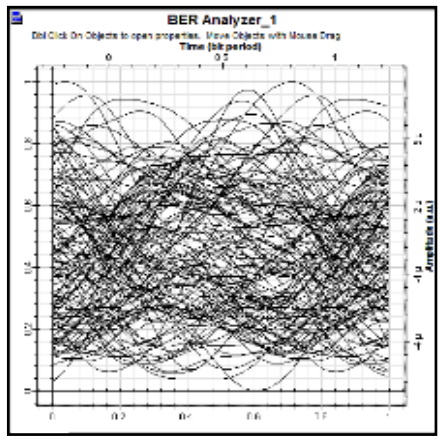

(a)

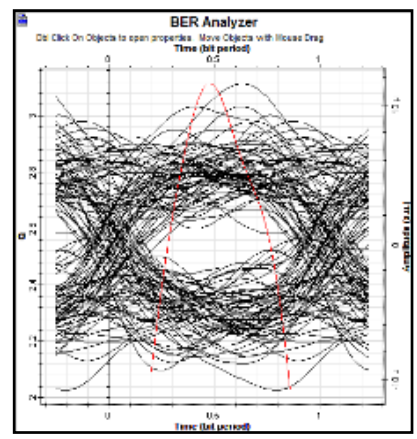

(d)

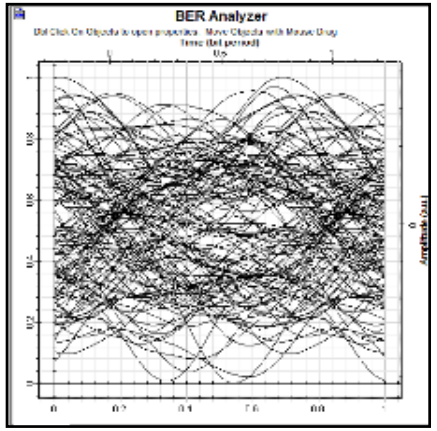

(b)

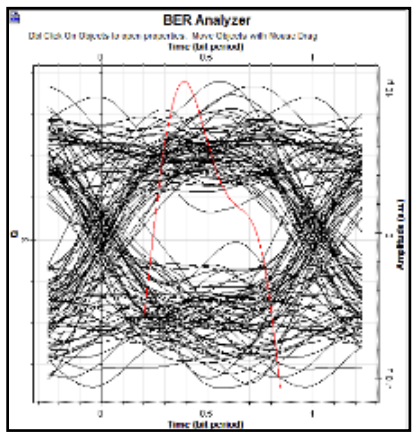

(c)

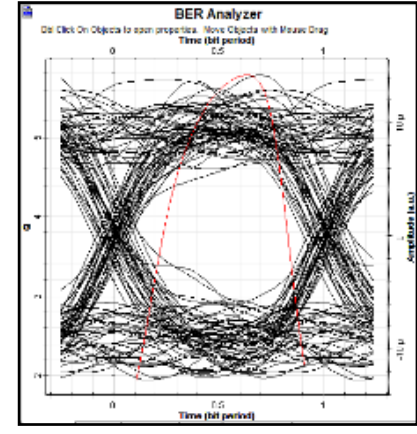

(e)

Figure 10. Eye pattern in 50cm aperture receiver for (a) Subtractor detection (b) SDD detection (c) Complimentary detection (d) AND detection (e) WFD detection

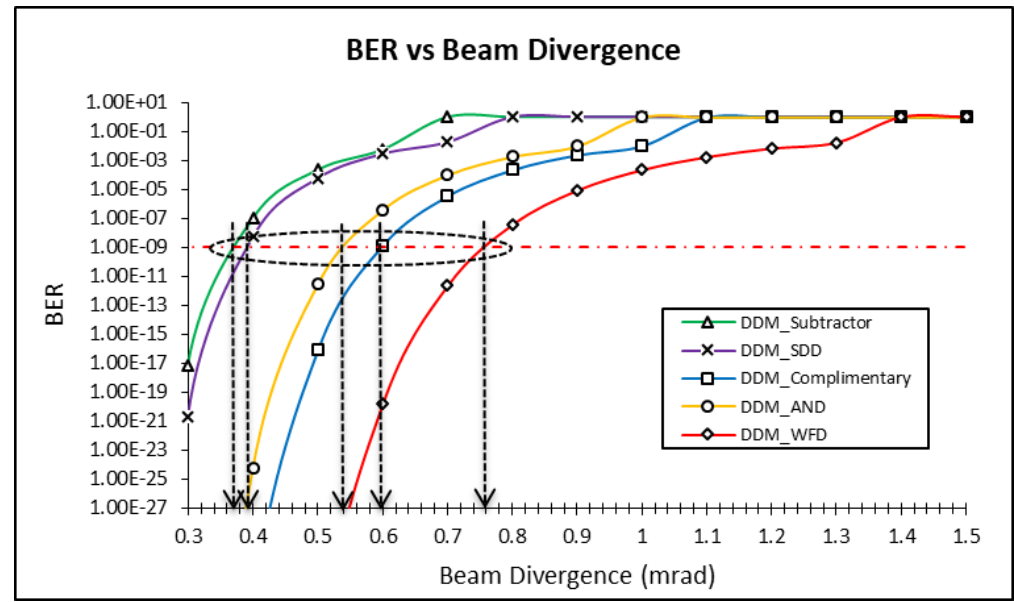

Figure 11. Bit error rate versus beam divergence for selection receiver detection 


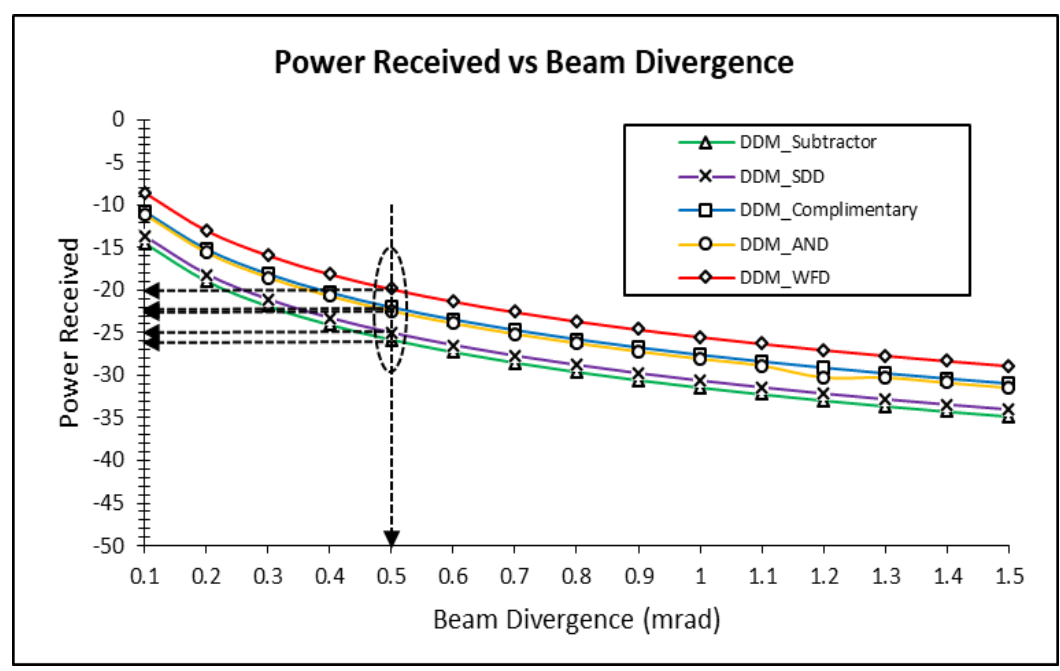

Figure 12. Power received versus beam divergence for selection receiver detection

\section{CONCLUSION}

In this paper, the analysis of receiver detection based on Dual Diffuser Modulation technique in FSO system have been determined. Here the analysis has been simulated over a specific aperture receiver and beam divergence in terms of error rate and power received. it has been concluded that DDM_WFD can enhance the system performance compare to the other receiver detection. The performance of DDM_WFD in acceptable bit error rate 10-9 shows that the performance increase 50\% compare to the existing detection. The power received in a high data rate 10Gbps makes DDM_WFD can perform well to transfer the signal. This detection technique ability can increase the power received $6 \mathrm{dBm}$ and the eye pattern is wide open potentially improve the overall system performance.

\section{ACKNOWLEDGEMENTS}

This project was supported by the Government of Malaysia under the Fundamental Research Grant Scheme at University Malaysia Perlis, Malaysia under the research grant \# 9003-00493.

\section{REFERENCES}

[1] Raj, I. D. A., \& Gokulakrishnan, K.:'Performance Measure of FSO by Modified AND Subtraction Method', International Journal of Scientific and Engineering Research, 2015, 3, (5), pp. 69-73.

[2] Al-Gailani, S. A., Mohammad, A. B., \& Shaddad, R. Q.:'Enhancement of Free Space Optical Link in Heavy Rain Attenuation using Multiple Beam Concept', Optik, 2013, 124, (21), pp. 4798-4801.

[3] Plank, T., Leitgeb, E., Pezzei, P., \& Ghassemlooy, Z.:'Wavelength-Selection for High Data Rate Free Space Optics (FSO) in Next Generation Wireless Communications'. 17th European Conference on Networks and Optical Communications, 2012, pp. 1-5.

[4] D. Killinger, 'Free Space Optics for Laser Communication through the Air', Optics and Photonics News, 2002, pp. 36-42.

[5] 'Optiwave Design Software for Photonics', http://www.optiwave.com, accessed 26 April 2016.

[6] Kadhim, D. S. A., Shakir, A. A. J., Mohammad, D. A. N., \& Mohammad4, N. F.:'System Design and Simulation using (OptiSystem 7.0) for Performance Characterization of the Free Space Optical', International Journal of Innovative Research in Science, Engineering and Technology, 2015, 4, (6), pp. 4823-2831.

[7] Chawla, A., Mishra, S., \& Aarthi, G.:'Simulation Research of Free-space Optical Communication Based on Linear Polarization Shift Keying Modulation', ARPN Journal of Engineering and Applied Sciences, 2015, 10, (14), pp. 6116-6120.

[8] Roy, R., \& Babu, J. S.:'Simulation and Performance Analysis of Free Space Optical Systems using Multiple TX / RX and Polarized CO-OFDM techniques under Atmospheric Disturbances', International Journal of Engineering Research and General Science, 2015, 3, (1), pp. 743-749.

[9] Rahman, A. K., Anuar, M. S., Nawawi, N. M., Aljunid, S. A., Rashidi, C. B. M., \& Junita, M. N.:'Enrich Data Bit Rate and Propagation Distance Performance for Free Space Optical Communication (FSOC) Via a New DualDiffuser Modulation (DDM) Technique'. 2nd International Conference on Computer, Communications, and Control Technology, Art Proceeding, 2015, pp. 341-345. 
[10] Rahman, A. K., Aljunid, S. A., Anuar, M. S., \& Fadhil, H. A.:'Improving Performance in Free Space Optical Communication (FSOC) Channel Through the Dual Diffuser Modulation (DDM) Due to Atmospheric Turbulence', Journal of Theoretical and Applied Information Technology, 2014, 60, (1), pp. 27-33.

[11] M. K. Abdullah, F. N. Hasoon, S. A. Aljunid, and S. Shaari, "Performance of OCDMA systems with new spectral direct detection (SDD) technique using enhanced double weight (EDW) code," Optics Communication, 2008, 281, (18), pp. 4658-4662.

[12] V. Nath, N. Jain, and S. Dogra, "Effect of Fiber Distance on Various SAC-OCDMA Detection Techniques," International Journal of Research in Engineering and Technology, 2013, 2, (3), pp. 290-293.

[13] R. Sahbudin, S. Aljunid, M. Abdullah, M. B. A. Samad, M. Mahdi, and M. Ismail, "Comparative Performance of Hybrid SCM SAC- OCDMA System Using Complementary and AND Subtraction Detection Techniques," The International Arab Journal of Information Technology (IAJIT), 2006, 5, (1), pp. 61-65.

[14] H. M. R. Al-khafaji, S. A. Aljunid, A. Amphawan, and H. A. Fadhil, "Triple-Play Services Using Different Detection Techniques for SAC-OCDMA Systems," 2012 IEEE 3rd International Conference on Photonics, 2012, pp. 350-354.

[15] I. Rao and D. Rana, "Comparison of Different Detection Techniques Based on Enhanced Double Weight Code in Optical Code Division Multiple Access System," International Conference of Advance Research and Innovation, (2015), pp. 126-132.

\section{BIOGRAPHIES OF AUTHORS}

M. F. Talib received B.Eng. (Hons) degree in Mechatronic Engineering form Universiti
Malaysia Perlis (UniMAP), Perlis, Malaysia in 2015. Currently, he is pursuing his study in
Master of Communication Engineering in UniMAP. His research focuses on Optical
Communication System.

